Bull. Mater. Sci., Vol. 35, No. 6, November 2012, pp. 997-1002. (c) Indian Academy of Sciences.

\title{
Influence of solvents on properties of solar selective coatings obtained by spray pyrolysis
}

\author{
MIHAELA DUDITA*, LUMINITA ISAC and ANCA DUTA \\ Renewable Energy Systems and Recycling Department, Transilvania University of Brasov, Eroilor 29, \\ 500036 Brasov, Romania
}

MS received 9 September 2011; revised 10 January 2012

\begin{abstract}
Solar selective coatings for solar thermal flat-plate collectors consisting of crystalline copper oxides and amorphous nickel oxide composites were obtained by robotic spray pyrolyzed deposition. The parameters were optimized for increased spectral selectivity $(S)$ : high solar absorptance and low thermal emittance. The coatings were deposited using nickel and copper acetate, dissolved in mixed solvents with various water: ethanol ratios. The coatings' properties were characterized in terms of crystalline composition (XRD), surface morphology (AFM, contact angle) and optical properties (solar absorptance, thermal emittance and spectral selectivity). Considering the precursor solutions composition (solvent, wetting behaviour), the growth processes were modelled for two different systems: predominant hydrophilic and predominant hydrophobic. The high selectivity values $(S>30)$ of the optimized composite coatings were explained based on two parallel mechanisms: intrinsic absorption and multiple reflections generated when absorbers with controlled roughness are deposited.
\end{abstract}

Keywords. Spray pyrolysis deposition; surface engineering; solar selective coatings.

\section{Introduction}

The active component in a solar thermal collector is the absorber plate which strongly influences the solar thermal conversion. Its role is to convert the incident solar radiation into heat, without significant heat losses (due to thermal radiation). Therefore, the absorber coating must have high solar absorption $\left(\alpha_{\mathrm{s}}>0.92\right)$ in the visible wavelength range $(0.29-2.5 \mu \mathrm{m})$ and low thermal emittance $\left(\varepsilon_{\mathrm{T}}<0.15\right)$ in the infrared spectral domain $(\lambda>2.5 \mu \mathrm{m})$ (Carlsson 2007). The ratio $\alpha_{\mathrm{s}} / \varepsilon_{\mathrm{T}}$ defines the spectral selectivity (Duffie and Beckman 2006).

The selective coatings evolved from the electrochemically deposited black chromium (Bayati et al 2005) or nickelpigmented alumina (Boström et al 2008; Zemanová et al 2008) to the coatings obtained by physical vapour deposition (PVD) (Nunes et al 2003) or chemical vapour deposition (CVD) (Oelhafen and Schüler 2005) which are mainly based on titanium oxynitride. These advanced coatings show good optical properties; however, the deposition processes require complex and high cost equipment and provide a rather poor ecological balance and energy pay-back time.

Spray pyrolysis deposition (SPD) involves standard equipment, a small amount of chemicals which leads to non-toxic byproducts, and is up-scalable for industrial applications, allowing large deposition surfaces (OjaAcik et al 2009).

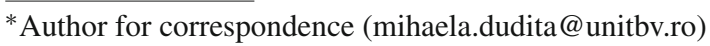

Nickel and copper oxide are widely used as multifunctional materials due to their good chemical stability, along with suitable electrical, electrochemical and optical properties. They have numerous applications ranging from electrochromic display devices (Valyukh et al 2010), sensors (Hongbing et al 2010), batteries (Souza et al 2006) to solar absorbers (Isac et al 2007). The use of single layer of black copper/nickel films based on copper oxides and nickel oxides for solar coatings was reported earlier (Voinea et al 2009). Statistic composites consisting of $\mathrm{CuO}_{x}$ and $\mathrm{NiO}_{x}$ are less studied, although increased spectral selectivity could be expected in this case.

The coatings surface morphology and roughness plays an important role in improving the absorber efficiency; textured/rough surface can favour multiple internal reflections leading to increased values of solar absorption. By using SPD, the films' morphology can be technologically controlled through the deposition parameters and precursor solution properties. The surface tension of the liquid influences the droplets formation and the wetting behaviour on the substrate and thus, the grain size and films' characteristics (surface roughness).

The paper focuses on the preparation of statistic composites, based on nickel and copper oxides with controlled optical and surface properties, to be used as selective coatings. The films were deposited from aqueous-alcoholic precursor solutions onto copper substrates by robotic spray pyrolysis. The influence of alcohol (ethanol) concentration on thin film formation mechanism and properties of films was investigated. 


\section{Experimental}

\subsection{Solar absorbers deposition}

Copper oxides and nickel oxides mixed films (samples $A 0-A 5)$ were deposited on copper substrate $(50 \times 50 \mathrm{~mm}$, Beofon), at $330{ }^{\circ} \mathrm{C}$, by robotic spray pyrolysis, from a previously optimized receipt (Voinea et al 2009) for single layer deposition. The technological parameters were previously optimized in order to obtain an optimum thickness of the layers $(800 \mathrm{~nm})$ considering the application and functional key parameter: spectral selectivity.

The substrate was mechanically polished and then washed with bidistillated water. A mixture of nickel acetate, $0.2 \mathrm{M}$ and copper acetate, $0 \cdot 1 \mathrm{M}$ (reagent grade, Scharlau), dissolved in water (W) and ethanol (Et), with alcohol concen-

Table 1. Precursor solution properties.

\begin{tabular}{|c|c|c|c|c|c|c|}
\hline Sample & $A 0$ & $A 1$ & $A 2$ & $A 3$ & $A 4$ & $A 5$ \\
\hline Solvent, Et\% & 0 & $2 \cdot 5$ & 5 & 10 & 20 & 40 \\
\hline$\sigma_{\text {p.s. }}(\mathrm{mN} / \mathrm{m})^{*}$ & $72 \cdot 4$ & 65 & $58 \cdot 9$ & $52 \cdot 2$ & $41 \cdot 1$ & $31 \cdot 2$ \\
\hline$\theta_{\mathrm{Cu}}(\text { degree })^{* *}$ & $73 \cdot 5$ & 73 & 69 & 67 & 55 & 40 \\
\hline
\end{tabular}

${ }^{*} \sigma_{\text {p.s. }}$ is the precursor solution surface tension;

${ }^{* *} \theta_{\mathrm{Cu}}$ represents contact angle measured for clean copper substrate and precursor solution. tration varying from 0 to $40 \%$, was used, as presented in table 1 .

\subsection{Solar absorbers characterization}

The crystalline composition was assessed using a Bruker D8 Discover Advanced Diffractometer using $\mathrm{CuK} \alpha$ radiation source $\left(\lambda=1 \cdot 5406 \cdot 10^{-10} \mathrm{~m}\right)$, in the range of $2 \theta=$ $2-80^{\circ}$. The surface morphology was evaluated by Atomic Force Microscope (AFM, NT-MDT model BL222RNTE). The images were taken in a semi-contact mode with Si-tips (NSG10, force constant $0 \cdot 15 \mathrm{~N} / \mathrm{m}$, tip radius, $10 \mathrm{~nm}$ ).

Static contact angle measurements for clean copper substrate and for the $A 0-A 5$ samples were performed with the sessile drop method, using an OCA-20 Contact Angle-meter

Table 2. Crystallite size dimensions.

\begin{tabular}{lccc}
\hline Sample & Solvent $\% \mathrm{Et}^{*}$ & $d_{\mathrm{CuO}}[\mathrm{nm}]$ & $d_{\mathrm{Cu}_{2} \mathrm{O}}[\mathrm{nm}]$ \\
\hline$A 0$ & 0 & $6 \cdot 7$ & $7 \cdot 4$ \\
$A 1$ & $2 \cdot 5$ & $10 \cdot 5$ & $4 \cdot 9$ \\
$A 2$ & 5 & $5 \cdot 56$ & 4.4 \\
$A 3$ & 10 & 14.9 & $6 \cdot 5$ \\
$A 4$ & 20 & 14.9 & 6.5 \\
$A 5$ & 40 & 10 & 8.6 \\
\hline
\end{tabular}

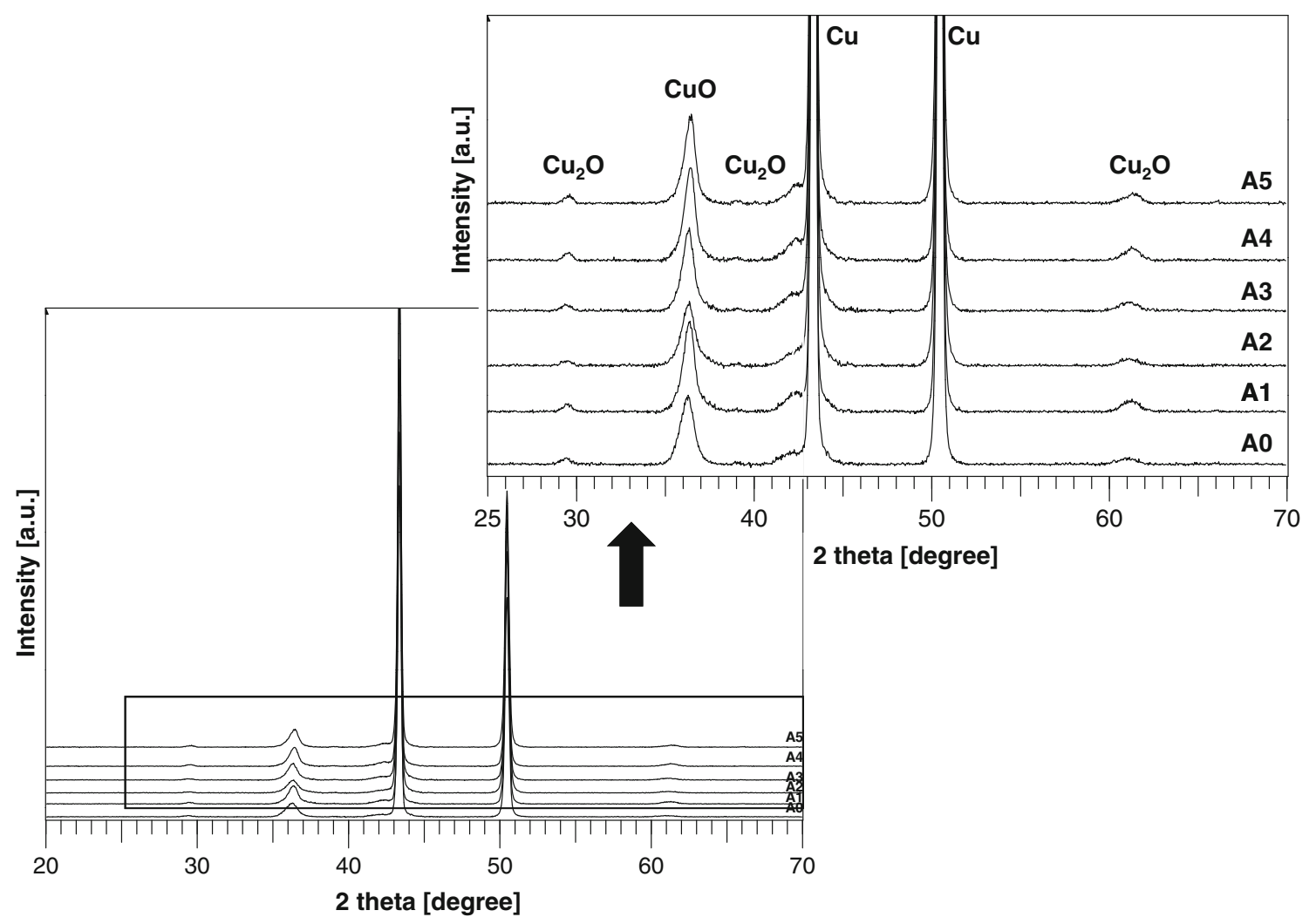

Figure 1. XRD patterns of films. 
(DataPhysics Instruments). A drop $(5 \mu \mathrm{L})$ of liquid-water or precursors solution-was placed, with $1 \mu \mathrm{L} / \mathrm{s}$ velocity, on the film's surface.
Optical absorption measurements were carried out with a UV-Vis Spectrophotometer (Perkin Elmer Lambda 25) and a FTIR Spectrophotometer (BXII Perkin Elmer). The solar
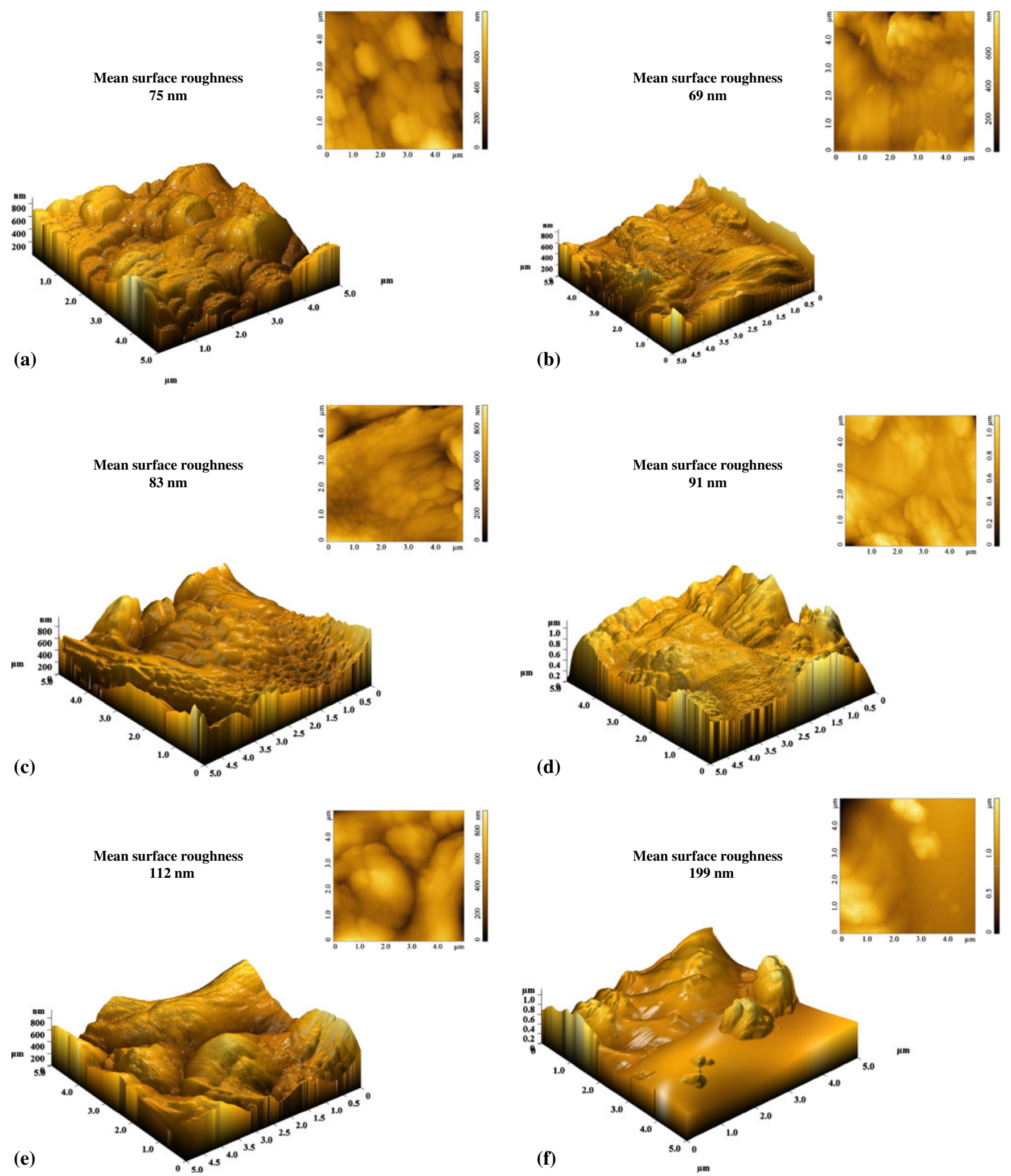

Figure 2. a-f. AFM image of sample obtained from precursor solutions with different alcohol concentrations: sample $A 0,0 \% \mathrm{Et}(\mathbf{a}), A 1$, $2.5 \%$ Et (b), A2-5 \% Et (c), A3-10\% Et (d), A4-20\% Et (e) and $A 5-40 \%$ Et (f). 
absorptance and thermal emittance were calculated from the reflectance spectra.

\section{Results and discussion}

$\mathrm{X}$-ray diffractograms of the samples obtained from aqueous and aqueous-ethanol solutions are presented in figure 1. Specific diffraction lines corresponding to $\mathrm{CuO}$ (tenorite, $\mathrm{PDF}$ 00-003-0884) and $\mathrm{Cu}_{2} \mathrm{O}$ (cuprite, PDF 00-003-0892) crystalline phases, together with $\mathrm{Cu}$ (PDF 01-071-3761) substrate, are identified. According to XRD patterns, no diffraction peaks for crystalline nickel oxides are observed. Based on preliminary studies for nickel ion identification and considering XRD diffraction patterns, at the deposition temperature, the nickel oxides are considered to be amorphous phases (Souza et al 2006).

The average crystallite sizes were calculated using the Scherrer formula (table 2). The solvent composition in the precursor solutions has no significant effect on the crystalline composition in thin films; the ethanol addition generally leads to the increase of the $\mathrm{Cu}_{2} \mathrm{O}$ average crystallite sizes and to a non-uniform variation of $\mathrm{CuO}$ average crystallite sizes. These variations have an influence on the films growth mechanism.

In developing a material for an efficient solar thermal absorber, the surface morphology is essential. Using atomic force microscopy, the sample morphologies were investigated and they are presented in figures $2 \mathrm{a}-\mathrm{f}$.

The AFM images show different surface morphologies, significantly depending on the ethanol content in the precursor solution. The sample $A 0$ (no ethanol addition) has a rather uniform, spherical morphology (figure 2a). As the concentration of alcohol increases, growth of the specified grains is promoted and aggregates are formed from

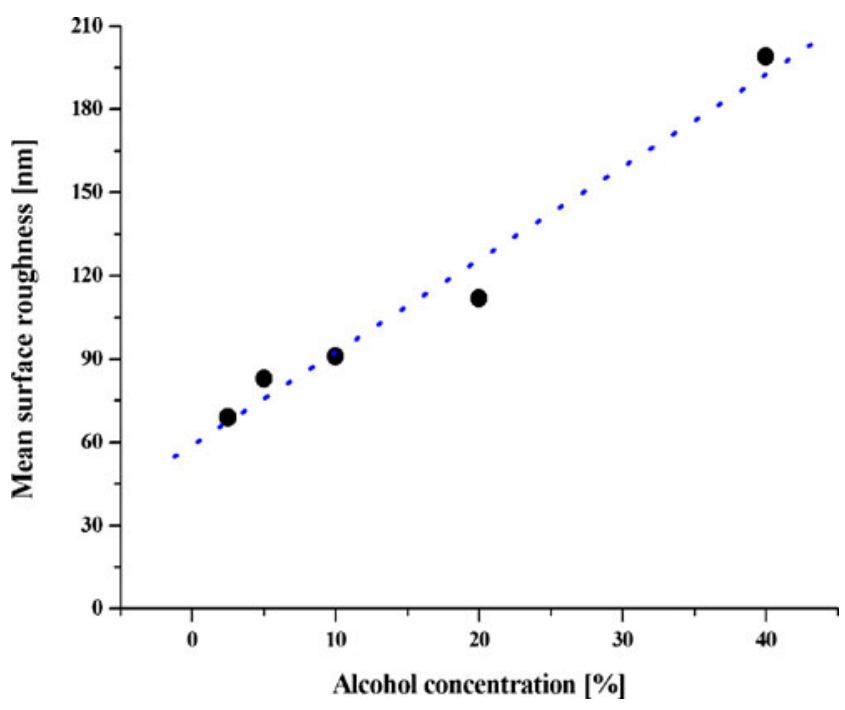

Figure 3. Variation of surface average roughness with ethanol content.
Table 3. Correlation of surface properties with spectral selectivity.

\begin{tabular}{lccccl}
\hline Sample & Solvent \% Et* & $\theta_{\mathrm{w}}[$ degree] & $\alpha_{\mathrm{s}}$ & $\varepsilon_{\mathrm{T}}$ & \multicolumn{1}{c}{$S$} \\
\hline$A 0$ & 0 & 52.5 & 0.915 & 0.030 & $30 \cdot 5$ \\
$A 1$ & $2 \cdot 5$ & $51 \cdot 0$ & 0.917 & $0 \cdot 030$ & $30 \cdot 6$ \\
$A 2$ & 5 & 38.5 & 0.919 & 0.075 & $12 \cdot 3$ \\
$A 3$ & 10 & $30 \cdot 2$ & 0.917 & 0.034 & 27 \\
$A 4$ & 20 & $20 \cdot 0$ & 0.917 & 0.030 & $30 \cdot 6$ \\
$A 5$ & 40 & $13 \cdot 5$ & 0.911 & 0.025 & $36 \cdot 4$ \\
\hline
\end{tabular}

*Percentage (in volume) of ethanol in water-ethanol precursors solution.

smaller crystallites/grains which merged into larger ones (figures $2 \mathrm{~b}-\mathrm{f}$ ). The consequence is aggregates sharpening and shallow cavities forming a phenomenon being known as "Ostwald ripening" (Validžić et al 2008). Following this mechanism, the surface roughness is also increasing with ethanol concentration in the precursor solution.

In the systems containing ethanol, a linear increase of the surface average roughness with ethanol content is registered, supporting the idea of a change in the nucleation/growth mechanism as a result of ethanol addition (figure 3). Considering the polarity difference between water and ethanol, the data suggest that there is a significant change in the cation (copper, nickel) hydration, thus in their reactivity.

Contact angle (CA) measurement is a simple and low cost method to assess the surface properties of a thin film, mainly the surface roughness/porosity, surface energy and wettability (Voinea et al 2008). Especially for solar absorbers, the wettability is very important: the final absorber surface must be hydrophobic (thus high contact angle measured for water, $>90^{\circ}$ ), while the intermediate layers in the case of multi-layer absorbers should be hydrophilic for water based precursors (with contact angle values lower than $90^{\circ}$ ).

The initial values of the contact angle measured for water range between 52.5 and 13.5 (table 3), indicating the formation of the hydrophilic surface required for a further deposition of the anti-reflective layer. There is a good correlation between surface roughness $\left(S_{\mathrm{a}}\right)$ and initial values of CA. As the samples have almost the same crystalline composition, CA values are rather influenced by the surface roughness. Thus, it is likely that the enhancement of the surface hydrophilic properties could be realized by adequately increasing the surface roughness.

It also has to be mentioned that the contact angle decrease has an opposite trend compared to roughness, but there is not a linear dependence; therefore, we may recommend contact angle measurements only as a preliminary and rough step in evaluating surface morphology evaluation.

An efficient solar selective coating has the solar absorption coefficient, $\alpha_{\mathrm{s}}>0.92$ and thermal emittance, $\varepsilon_{\mathrm{T}}<$ $0 \cdot 15$ (Carlsson 2007). The absorbers reported in this paper have a very good spectral selectivity, as the data presented in table 3 show. The high solar absorption values can 
be explained by the following mechanisms (Bayati et al 2010):

- intrinsic absorption or normal absorption; when light propagates through a medium that is not vacuum, it attenuates, which is a fundamental property for a solar coating;

- multiple reflection/absorption: when rough absorbers are used, the waves are subjected to multiple reflection into the narrow(er) pores of the layer and the waves' energy is partially absorbed into the material for each contact, leading to an increased solar absorptivity.

\subsection{Growth mechanism}

During the spray pyrolysis, small droplets are formed from the precursor solutions. The metal acetate molecules are thermally cracked, and several reactions occur like dehydration ((1) and (2)) followed by decomposition ((3) and (4)), leading to nucleation and thin film growth.

$$
\begin{aligned}
& \mathrm{Cu}\left(\mathrm{CH}_{3} \mathrm{COO}\right)_{2} \cdot \mathrm{H}_{2} \mathrm{O} \underset{-\mathrm{H}_{2} \mathrm{O}}{\longrightarrow} \mathrm{Cu}\left(\mathrm{CH}_{3} \mathrm{COO}\right)_{2}, \\
& \mathrm{Ni}\left(\mathrm{CH}_{3} \mathrm{COO}\right)_{2} \cdot 4 \mathrm{H}_{2} \mathrm{O} \underset{-4 \mathrm{H}_{2} \mathrm{O}}{\longrightarrow} \mathrm{Ni}\left(\mathrm{CH}_{3} \mathrm{COO}\right)_{2}, \\
& \mathrm{Cu}\left(\mathrm{CH}_{3} \mathrm{COO}\right)_{2}+4 \mathrm{O}_{2} \rightarrow \mathrm{CuO}+4 \mathrm{CO}_{2}+3 \mathrm{H}_{2} \mathrm{O}, \\
& \mathrm{Ni}\left(\mathrm{CH}_{3} \mathrm{COO}\right)_{2}+4 \mathrm{O}_{2} \rightarrow \mathrm{NiO}+4 \mathrm{CO}_{2}+3 \mathrm{H}_{2} \mathrm{O} .
\end{aligned}
$$

During SPD, droplets size in the aerosol has an important role in film formation. The droplets diameter is directly influenced by the precursor solution surface tension; the direct proportionality between the pressure difference (internal and external droplet pressure) and the surface tension is given by the well-known Young-Laplace equation. Thus, decreasing the surface tension (by adding ethanol) results in lower internal pressures and/or in the possibility of getting lower sized droplets. This can explain the roughness increase while developing larger aggregates. Supplementarily, the contact angles were measured for each precursor solution in contact with clean copper substrate (table 1) which show an increased wetting behaviour of the precursor solution with increasing alcohol concentration.

Alcohols are known to reduce the surface tension of the water based solutions. The precursor drops will generate a less isolated nucleation centre due to the formation of large particles which tend to coalesce, for reducing their surface energy. Further, particle growth might be a result of continuing nucleation in the film on the substrate, and Ostwald ripening type of growth will lead to the formation of large oriented aggregates (Singh et al 2007).

Based on the Murakami et al (2007) mechanism, the film growth was modelled (figure 4) considering: (a) nucleation and (b) thin film growth and/or coalescence

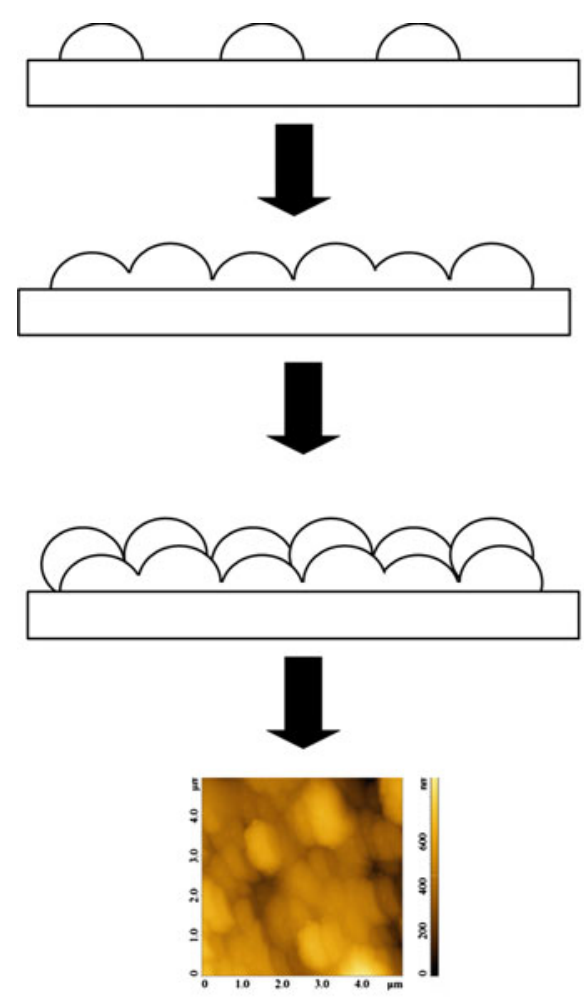

Surface roughness $=75 \mathrm{~nm}$
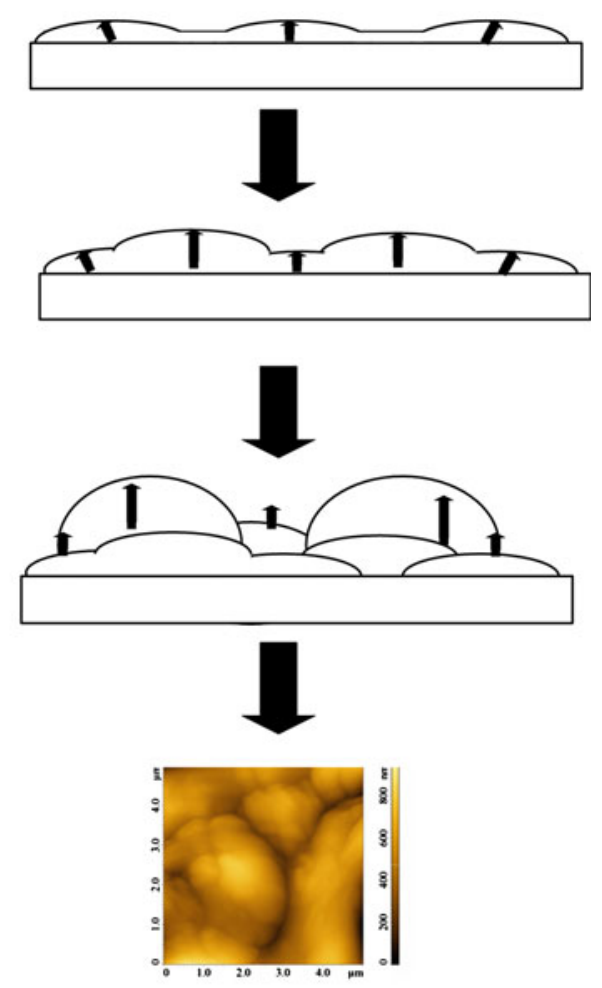

Surface roughness $=112 \mathrm{~nm}$

(a)

(b)

Figure 4. Mechanism proposed for films deposition by SPD from precursor solutions with: (a) high contact angle and (b) low contact angle, measured on copper substrate. 
growth. Two different precursors were considered: solutions with good wetting behaviour-as in the case of alcoholic precursor solutions-and average wetting solutions. First, isolated nuclei are formed on the heated substrate. In the case of films obtained from solutions with higher alcohol content, thus low contact angle, the number of isolated nuclei is rather small and the growth rate is predominant. The grains can further coalesce to form larger aggregates. Therefore, the grain size and also the surface roughness increases. In this case, particle growth might be a result of an Ostwald ripening growth type and it can occur mainly through solid-state diffusion. For the solutions with low wetting behaviour, the particle growth is mainly due to continuous nucleation with the formation of rather small spherical particles (figure 4).

\section{Conclusions}

Selective coating based on copper and nickel oxides statistic composites with controlled optical and surface properties were deposited from aqueous-alcoholic mixed precursor solutions onto copper substrates, by robotic spray pyrolysis. The method involves a small amount of chemicals which leads to non-toxic byproducts and can be used in industrial applications.

The alcohol percentage is modifying the surface tension of the precursor solution which influences the droplets formation and thus the film characteristics (surface roughness).

The solar absorbers have high selectivity values $(S>30)$ as a result of the intrinsic absorption due to the presence of copper and nickel oxides, and due to multiple reflections generated when rough enough absorbers are used. The best selectivity (36.4) is registered for thin films with highest roughness, obtained from precursors with $40 \%$ ethanol, in the water-ethanol mixed solvent.

Based on the main precursor characteristics and morphology studies, a growth mechanism is proposed for two different precursor solutions: with good wetting behaviour as in the case of alcoholic precursor solutions and with average wetting solutions.

Modifying the alcohol concentration in the precursor solutions and the wetting behaviour of the precursor solution represents an efficient path to change growth processes of thin film, especially the surface properties (surface roughness) and, consequently the absorber's spectral selectivity.

\section{Acknowledgements}

This paper is supported by the Sectoral Operational Programme Human Resources Development (SOP HRD) Post-Doctoral Studies, financed from the European Social Fund and by the Romanian Government under contract number, POSDRU 59323.

\section{References}

Bayati M R, Shariat M H and Janghorban K 2005 Renew. Energ. 302163

Bayati M R, Zargar H R, Talimian A, Ziaee A and Molaei R 2010 Surf. Coat. Technol. 2052483

Boström T, Jensen J, Valizadeh S, Westin G and Wäckelgård E 2008 Sol. Energ. Mat. Sol. C. 921177

Carlsson B 2007 Sol. Energ. Mat. Sol. C. 911338

Duffie J A and Beckman W A 2006 Solar engineering of thermal processes (New Jersey: John Wiley \& Sons) 3rd ed., pp 175-201

Hongbing W, Hongwen S, Sumei W, Guangzhi C, Hou H, Hongwen G and Xiaodong M 2010 J. Nat. Gas Chem. 19393

Isac L, Duta A, Kriza A, Manolache S and Nanu M 2007 Thin Solid Films $\mathbf{5 1 5} 5755$

Murakami K, Nakajima K and Kaneko S 2007 Thin Solid Films 515 8632

Nunes C, Teixeira V, Prates M L, Barradas N P and Sequeira A D 2003 Thin Solid Films 442173

Oelhafen P and Schüler A 2005 Sol. Energ. 79110

OjaAcik, Junolainen A, Mikli V, Danilson M and Krunks M 2009 Appl. Surf. Sci. 2561391

Singh P, Kumar A, Deepak and Kaur D 2007 J. Cryst. Growth 306303

Souza E A, Landers R, Cardoso L P, Cruz T G S, Tabacniks M H and Gorenstein A 2006 J. Power Sources 155358

Validžić I L, Jokanović V, Uskoković D P and Nedeljković J M 2008 Mater. Chem. Phys. 10728

Valyukh I, Green S, Arwin H, Niklasson G A, Wäckelgård E and Granqvist C G 2010 Sol. Energ. Mat. Sol. C. 94724

Voinea M, Vladuta C, Bogatu C and Duta A 2008 Mater. Sci. Eng. $B-A d v .15276$

Voinea M, Ienei E, Bogatu C, Chitanu G C and Duta A 2009 J. Nanosci. Nanotechnol. 94279

Zemanová M, Chovancová M, Gáliková Z and Krivošík P 2008 Renew. Energ. 332303 